\title{
Correction: Cancer-associated fibroblasts: overview, progress, challenges, and directions
}

\author{
Qinrong Ping $\cdot$ Ruping Yan - Xin Cheng - Wenju Wang - Yiming Zhong - Zongliu Hou - Yunqiang Shi · \\ Chunhui Wang 1 . Ruhong Li 1
}

Published online: 28 June 2021

(c) The Author(s), under exclusive licence to Springer Nature America, Inc. 2021

\section{Correction to: Cancer Gene Therapy \\ https://doi.org/10.1038/s41417-021-00318-4}

The article "Cancer-associated fibroblasts: overview, progress, challenges, and directions", written by Qinrong Ping, Ruping Yan, Xin Cheng, Wenju Wang, Yiming Zhong, Zongliu Hou, Yunqiang Shi, Chunhui Wang \& Ruhong $\mathrm{Li}$, was originally published online on the publisher's internet portal on 12 March 2021 with Open Access under a Creative Commons Attribution 4.0 International License.

With the author's/authors' decision to cancel Open Access the copyright of the article changed on 28 April 2021 to (C) The Author(s), under exclusive licence to Springer Nature America, Inc. 2021 with all rights reserved. 